\title{
A Framework for Analysis of Connectivity and Performance Bounds in Ad Hoc Networks and Its Application to a Slotted-ALOHA Scenario
}

\author{
Fabrizio Granelli, Member, IEEE
}

\begin{abstract}
In this paper, a framework is proposed to analyse the problems of connectivity and performance in ad-hoc networks through an analytical approach. To this aim, available results regarding the application of percolation theory to the study of connectivity in ad-hoc networks are exploited jointly with communication theory models in order to derive the configuration of network parameters that ensures long range connectivity among nodes and the corresponding available capacity on the wireless medium. The framework is then applied to a slotted ALOHA ad-hoc network. Theoretical and numerical results validate the approach and allow the derivation of interesting design principles for ad-hoc networks that consider the impact of physical and MAC-level parameters on network connectivity and end-to-end performance.
\end{abstract}

Index terms: ad-hoc networks, performance evaluation, slottedALOHA

\section{INTRODUCTION}

Mobile ad-hoc networks (MANET) consist of wireless hosts that communicate with each other in the absence of a fixed infrastructure [8]. They can be used in a wide plethora of applications, ranging from tactical operations, to establish quickly military communications during the deployment of forces in unknown and hostile terrain; to sensor networks, for communication between intelligent sensors mounted on mobile platforms. In the last application, mobile ad hoc networks are likely to achieve wide deployment in the near future because they greatly extend the ability to monitor and control the physical environment from remote locations.

In ad-hoc wireless networks, effective exploitation of the shared transmission resources and power control are two key elements representing research challenges. Not all hosts are in fact within the transmission range of each other and communication is achieved by multi-hop routing, where intermediate nodes cooperate by forwarding packets between two hosts. Available bandwidth on the wireless medium is partitioned / shared by all the nodes in a given range and access to the medium is usually performed in a distributed fashion.

Moreover, employment of batteries in ad-hoc networks nodes imposes strong constraints on energy consumption and

Manuscript received May 31, 2004; revised May 09, 2005 and July 21, 2005. The paper was presented in part at the Conference on Software, Telecommunications and Computer Networks (SoftCOM) 2004.

F. Granelli is with DIT - University of Trento, I-38050 Trento, Italy (e-mail: fabrizio.granelli@unitn.it). trasmission power. Key point for the existence of the network is in fact the life of each node, which should be maximized, and thus energy saving.

At the present moment, a relevant scientific effort is devoted to the design and implementation of ad-hoc networks, and in particular the development of useful models as tools for comparing different design principles or solutions represents an open issue.

In such framework, the usage of percolation theory using a physical model for transmission based on the signal to noise ratio was recently proposed [1] for studying capacity and connectivity issues in ad hoc networks in presence of interference. An interesting approach is presented in [2], which considers an extension of the model to the case where interference among contemporary nodes transmission is mitigated through the introduction of a constant $(\gamma)$ to include the employment of orthogonal CDMA codes, as a way to effectively reduce the impact of interfering signals.

The present work focuses on the issues related to resource and power allocation / management in ad-hoc networks, in order to provide guidelines for the design and configuration of ad-hoc networks under constraints of network connectivity and performance bounds of the resulting architecture.

From a methodological point-of-view, the paper represents a further generalization of the work presented in [2] by introducing a framework that considers in addition to physical parameters (transmission power, modulation parameters, etc.) the existence of the medium access control (MAC) protocol, that coordinates and manages access to the wireless medium. Different MAC strategies have a relevant impact on ad-hoc network connectivity and performance.

The framework is then applied in a scenario where the available bandwidth is shared among all nodes and the MAC protocol employs a slotted-ALOHA strategy. Results will analyse the configurations of parameters (node spatial density, transmission power, etc.) of the network that grant connectivity between two distant nodes.

The structure of the paper is as follows: section II introduces the framework and the proposed model, while the relevant theoretical results are derived in section III. Finally, section IV presents some numerical results and section $\mathrm{V}$ draws conclusions and outlines future work on the topic.

\section{THE PROPOSED APPROACH}

\section{A. The Proposed Framework}

The considered scenario is a multi-hop ad-hoc network of infinite extension, where nodes are spatially distributed 
according to a Poisson distribution of constant density $\lambda$, like in $[2] . \lambda$ represents the average number of nodes per square meter. Each node transmits using a power level $P_{i}$, selected in the range $\left[0, P_{t}\right]$ and tuned in such a way to reach its neighbors. $P_{t}$ is the maximum available transmission power of each node. Finally, $\mathbf{x}_{\mathbf{i}}$ is a vector representing the position of the $i$-th node (two-dimensional in the case considered throughout the paper), while $\mathrm{L}(\mathbf{x})$ is the attenuation function experienced by a signal on a distance equal to $\mathbf{x}$. L(x) depends from the specific propagation model under exam.

In case the access to the medium is perfectly scheduled (one transmission at a time, no interference - like in Time Division or Frequency Division Multiple Access), transmission between node $i$ and node $j$ is successful if the received signalto-noise ratio is higher than a threshold $\beta$ :

$$
\frac{P_{i} \cdot L\left(x_{i}-x_{j}\right)}{T_{n}} \geq \beta
$$

where $T_{n}$ is the thermal noise power, given by $F k T_{0} B$ ( $F$ is the noise figure, $k$ is the Boltzmann's constant, $B$ the transmission bandwidth and $T_{0}$ the temperature of the environment) [2]. Eq.(1) models the well-known principles of communication systems, that require a minimum ratio between received signal power and noise in order to allow effective decoding at the receiver side. The threshold $\beta$ depends on several design aspects: the employed modulation, the transmission / reception schemes, the usage of error correction schemes, etc.

In a more realistic scenario, other nodes will access the medium through the selected MAC strategy and thus potentially generating interference to the transmission between nodes $i$ and $j$. Therefore, we need to enhance Eq.(1) by introducing the contribution of the other active nodes and obtaining the following relationship:

$$
\frac{P_{i} \cdot L\left(x_{i}-x_{j}\right)}{T_{n}+\left(1-g_{M A C}\right) \cdot \sum_{\substack{\text { active } \\ \text { nodes }}} P_{k} \cdot L\left(x_{k}-x_{j}\right)} \geq \beta
$$

where $g_{M A C}$ is the MAC-level factor of merit of the network and represents a multiplicative term (between 0 and 1) that models the mitigation effects on interference of the specific MAC strategy employed for accessing the medium. It can be easily seen that for $g_{M A C}=1$ (perfect scheduling: TDMA, FDMA), Eq.(2) becomes Eq.(1).

It should be noticed that Eq.(2) contains both the constraints on the physical interference level and medium access control efficiency to achieve successful transmission on a given link.

\section{B. The Considered Scenario}

We restrict our analysis to the case of a perfect wireless channel (the effect of transmission on the channel is only attenuation), characterized by the following propagation model:

$$
L(x)=\|x\|^{-\alpha} \quad 3 \leq \alpha \leq 6
$$

A relevant aspect in the framework of ad-hoc networks is the Medium Access Control (MAC) protocol, since it defines the procedures for sharing the wireless medium common to all terminals belonging to the network. More in detail, access to communication resources in ad-hoc networks is based on two principles: resource sharing (the wireless medium is shared among nodes in a neighborhood defined on the basis of the transmission range) and space-division (interference is limited to neighboring nodes). Several approaches have been proposed so far, starting from ALOHA and CSMA (for generic radio communications) up to ad-hoc network specific protocols such as MACA [10], FAMA [11], MACAW [12], MACA-BI [13], etc. For a good, comprehensive and up-todate review of existing medium access control schemes for ad hoc networks and open challenges, the reader should refer to [9].

The remainder of the paper is focused on a Slotted-ALOHA medium access control strategy, because it is the simplest and least computational extensive, while maintaining the features of random access and distributed implementation of the modern schemes. Furthermore, the considered scenario allows to provide closed-form and easy-to-interpret solutions to the problem of planning ad-hoc networks. However, the framework remains valid also for other MAC schemes, given the possibility of estimating the term $g_{M A C}$ in Eq.(2).

In Slotted-ALOHA [4], the available resources are partitioned into equal-size time slots, and nodes are allowed to transmit in any slot without control - but only on the beginning of each time slot. If just one node accesses a given slot and no errors are introduced, transmission is successful and the receiver sends an acknowledgement back to the sender in order to confirm the data. ACKs are not considered in the paper, supposing they are very small and have a very low probability of interfering with other transmissions. The advantage of the approach is that - under specific circumstances which will be defined in the following sections - it provides a simple yet effective exploitation of the available resources, since in principle resources are assigned to the nodes that need them. In fact, in the case only one node access the medium in a given time slot, it gains full access to the available channel.

In order to evaluate $\mathrm{g}_{\mathrm{MAC}}$, the probability that a given station/node accesses the shared channel in a given slot is labeled $p$ (both slot time and $p$ are equal for any node). The probability that more than 1 node accesses the medium during a given slot (i.e. the probability of collisions) is then given by

$$
\operatorname{Pr}\{\text { collisions }\}=1-(1-p)^{N-1}
$$

where $N$ is the number of nodes in the local neighborhood (including node $i$ ). As a consequence, the nodes that can potentially interfere with the transmission of node $i$ is $N-1$.

Starting from Eq.(4), it is possible to write

$$
1-g_{M A C}=\left\lfloor 1-(1-p)^{N-1}\right\rfloor
$$

And finally, by substituting (5) in (2), we find an updated model for successful transmission, which contains a set of physical and MAC-level design parameters:

$$
\frac{P_{i} \cdot L\left(x_{i}-x_{j}\right)}{T_{n}+\left[1-(1-p)^{N-1}\right] \cdot \sum_{\substack{\text { active } \\ \text { nodes }}} P_{k} \cdot L\left(x_{k}-x_{j}\right)} \geq \beta
$$


Eq.(6) represents the constraint for successful transmission between node $i$ and node $j$ in the case of employing the slotted-ALOHA access discipline.

Since nodes are Poisson-distributed in the space with average $\lambda$, it is possible to estimate the maximum number of potentially interfering nodes by considering the ones within the maximum transmission range $r_{\max }$. From [2] it is known that, if $L(x)=\|x\|^{-\alpha}$, then - neglecting mutual intereference - it is possible to evaluate $r_{\max }$ as:

$$
r_{\max }=I_{m}\left(\frac{P_{t}}{\beta T_{n}}\right)^{1 / \alpha}
$$

where $I_{m}$ is the unitary distance costant (equal to 1 meter). As a consequence:

$$
N=\lambda \cdot \pi r_{\max }^{2}=\lambda \cdot \pi I_{m}^{2}\left(\frac{P_{t}}{\beta T_{n}}\right)^{2 / \alpha}
$$

\section{THEORETICAL RESULTS}

Eq.(6) is the same as the first condition presented in [2, Eq.(1)]. In fact, if we substitute $\gamma=1-(1-p)^{N-1}$, we obtain:

$$
\frac{P_{i} \cdot L\left(x_{i}-x_{j}\right)}{T_{n}+\gamma \cdot \sum_{\substack{\text { active } \\ \text { nodes }}} P_{k} \cdot L\left(x_{k}-x_{j}\right)} \geq \beta
$$

As a consequence, it is possible to exploit the results presented in [2] as a starting point and generalize for further analysis of connectivity problems in ad-hoc networks.

\section{A. Percolation}

The main result is that we can apply the percolation theory [3] to characterize connectivity of the ad-hoc network, i.e. to discriminate under which conditions the network is made of disconnected clusters of nodes or distant nodes are enabled to transfer data (full connectivity). More in details, we are interested in studying for which configurations of the network parameters it is theoretically possible to set up long range connections.

From results in [2] we know that:

$>$ A percolation threshold exists for $\gamma=0(p=0)$

$>$ A percolation threshold also exists for $\gamma<\gamma^{*}$, with $\gamma^{*}<1 / \beta$. In this case, the value of $\gamma^{*}$ can only be approximately determined through numerical simulations.

In our case, since the case $p=0$ is straightforward and could be considered as a reference case, we focus on the second case:

$$
\begin{aligned}
& \gamma=1-(1-p)^{N-1}<\gamma^{*} \\
& 1-p>\exp \left[\frac{\ln \left(1-\gamma^{*}\right)}{N-1}\right] \\
& p<1-\exp \left[\frac{\ln \left(1-\gamma^{*}\right)}{N-1}\right]=1-\exp \left[\frac{\ln \left(1-\gamma^{*}\right)}{\lambda \pi r_{\max }^{2}-1}\right]
\end{aligned}
$$

Eq.(12) confirms the empirical consideration that if $p$ is too large, then no long range communication is possible due to the high number of collisions on the wireless medium. By knowing it, it is possible to evaluate the critical probability of medium access by a single station, given by:

$$
p^{*}=1-\exp \left[\frac{\ln \left(1-\gamma^{*}\right)}{\lambda \pi r_{\text {max }}^{2}-1}\right]
$$

Another result is the critical node density above which connectivity is granted. In this case, starting from (12) it is possible to find that:

$$
\begin{aligned}
& \lambda \pi r_{\max }^{2}-1>\frac{\ln \left(1-\gamma^{*}\right)}{\ln (1-p)} \\
& \lambda>\frac{1}{\pi}\left(\frac{P_{t}}{\beta \cdot T_{n}}\right)^{-2 / \alpha} \cdot\left[1+\frac{\ln \left(1-\gamma^{*}\right)}{\ln (1-p)}\right] \\
& \lambda^{*}=\frac{1}{\pi}\left(\frac{P_{t}}{\beta \cdot T_{n}}\right)^{-2 / \alpha} \cdot\left[1+\frac{\ln \left(1-\gamma^{*}\right)}{\ln (1-p)}\right]
\end{aligned}
$$

Figure 1 depicts the behaviour of $p^{*}$ and $\lambda^{*}$ against the parameters of the network.

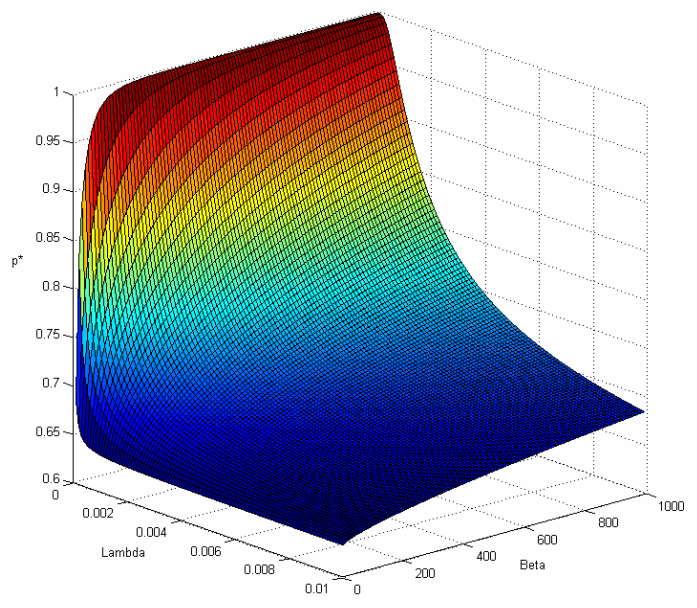

(a)

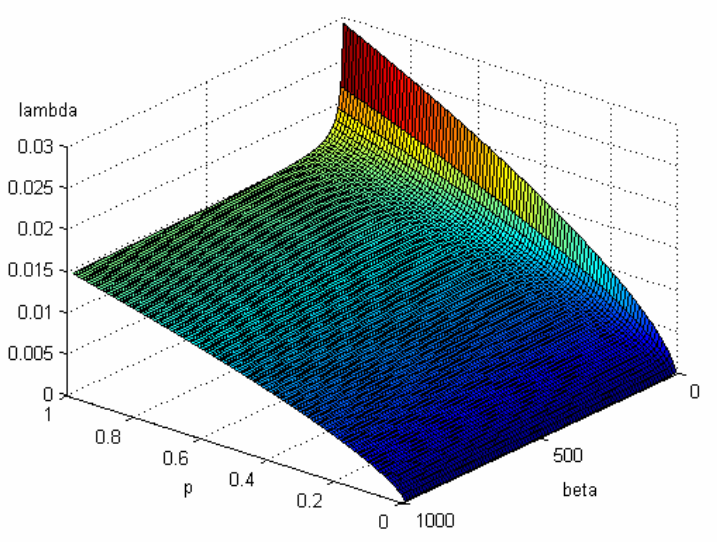

(b)

Fig. 1. Behaviour of $p^{*}$ (a) and $\lambda^{*}(b)$ in the $(\lambda, \beta)$ and $(p, \beta)$ spaces, respectively $\left(\mathrm{P}_{\mathrm{t}}=100 \mathrm{~mW}, \mathrm{~N}_{0}=10^{-6}, \alpha=3, \gamma^{*}=0.01\right)$.

\section{B. Connectivity}

The maximum number of nodes $N_{c}$ that a node can be locally connected is given by [2]: 


$$
\begin{aligned}
& N_{c} \leq 1+\frac{1}{\beta \cdot \gamma}=1+\frac{1}{\beta \cdot\left[1-(1-p)^{N-1}\right]} \\
& N_{c} \leq 1+\frac{1}{\beta \cdot\left[1-(1-p)^{\lambda \cdot \pi_{\max }^{2}-1}\right]}
\end{aligned}
$$

As a consequence, we can define the connection density $\delta$ as the product between the node density $\lambda$ and the maximum number of connections $N_{c}$ of a single node:

$$
\delta=\lambda \cdot N_{c} \quad\left[\frac{\text { connections }}{\text { square meter }}\right]
$$

The connection density represents the number of connections per unit area that can be supported by the considered ad-hoc network.

\section{Perfomance Bounds}

Recalling Hartley-Shannon's law for the capacity of a communication channel [5], the capacity $C$ of a channel can be calculated in on the basis of the available bandwidth $B$ and the signal-to-noise ratio at the receiver $S / N$ with the following formula:

$$
C=B \cdot \log (1+S / N)
$$

In the considered scenario, it is then possible to calculate the theoretical maximum capacity related to any of the links within a transmission path (end-to-end - E2E) as:

$$
C_{i, j}=B \cdot \log \left[1+\frac{P_{i} \cdot L\left(x_{i}-x_{j}\right)}{T_{n}+\left[1-(1-p)^{N-1}\right] \cdot \sum_{\substack{\text { active } \\ \text { nodes }}} P_{k} \cdot L\left(x_{k}-x_{j}\right)}\right]
$$

The total capacity over the entire transmission path is given by the minimum capacity on all the links. Considering the available end-to-end paths where Eq.(6) is verified on every point-to-point link, it is possible to evaluate the end-to-end capacity of any existing multi-hop path through the network:

$$
C_{E 2 E}=\min _{i, j}\left\{C_{i, j}\right\}>B \cdot \log (1+\beta)
$$

Eq.(22) defines the minimum channel capacity associated to any link among two nodes in the ad-hoc network that can be achieved in our scenario (in the case percolation is verified) and thus it represents the minimum capacity over an established end-to-end path through the ad-hoc network. This result is general, since it is valid as long as Eq.(6) holds.

Another interesting result is the possibility of computing an estimate of the end-to-end bit error rate (BER) by using a method similar to the one presented in [6]. In our scenario, considering a binary phase shift keying (BPSK) modulation:

$$
B E R_{E 2 E} \approx 1-\left[1-Q\left(\frac{P_{i} \cdot L\left(x_{i}-x_{j}\right)}{T_{n}+\left[1-g_{\text {MAC }}\right] \cdot \sum_{\substack{\text { active } \\ \text { nodes }}} \cdot L\left(x_{k}-x_{j}\right)}\right)\right]^{N_{h}}
$$

where $N_{h}$ is the number of hops over the end-to-end transmission path.

Again, it is possible to find a lower bound for BER $\mathrm{E} 2 \mathrm{E}_{\mathrm{E}}$ by using the constraint in Eq.(6):

$$
B E R_{E 2 E, \min } \approx 1-[1-Q(\beta)]^{N_{h}}
$$

Eq.(22) and (24) allows joint estimation of the channel bitrate and the BER performance on the end-to-end data connection in the considered wireless ad-hoc network. Knowing such information and the format of the MAC-level frame it is possible to derive the end-to-end frame error rate $\left(\mathrm{FER}_{\mathrm{E} 2 \mathrm{E}}\right)$. Furthermore, the transmission rate over the end-toend data path will be derived from (22) as

$$
R_{E 2 E}=\alpha_{M A C} \cdot \gamma_{P H Y} \cdot C_{E 2 E}
$$

where $\gamma_{\mathrm{PHY}}$ introduces sub-optimal performance (with respect to Hartley-Shannon law) due to physical level modulation / coding schemes, while $\alpha_{\text {MAC }}$ takes into account reduction in data rate due to the employed MAC strategy.

It must be underlined that the proposed framework provides useful results also in the case of different MAC strategies, as long as it remains possible to characterize the network behaviour cosistent with formula (2).

\section{NUMERICAL RESULTS AND DISCUSSION}

This section contains numerical results used for underlining some design principles for ad-hoc networks. First, numerical results are related to the problem of achieving full network connectivity. The employed algorithm (implemented in the Matlab environment [7]) is the following:

- the node topology is randomly generated using a Poissonian distribution with parameter $\lambda$;

- for each node, the potential interference deriving by the active nodes in that time slot is computed and the corresponding signal-to-noise ratio for any receiving node is computed;

- if the signal-to-noise is higher than $\beta$, then connection is available between the considered node couple.

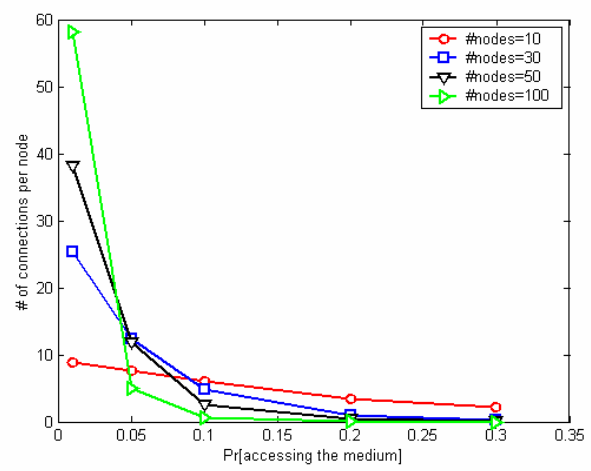

(a)

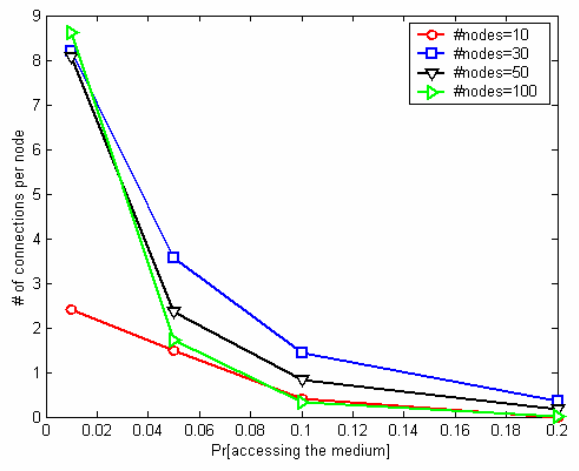

(b) 
Fig. 2. No. of connections per node against 'p': $\mathrm{P}_{\mathrm{t}}=10 \mathrm{~mW}(\mathrm{a})$,

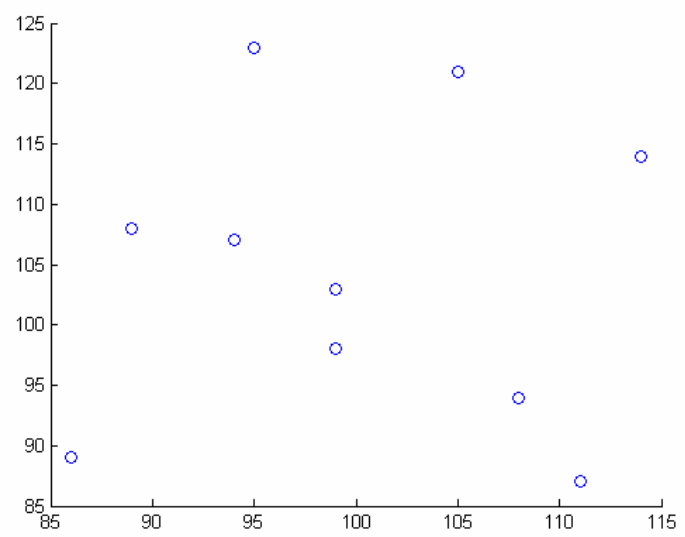

(a)

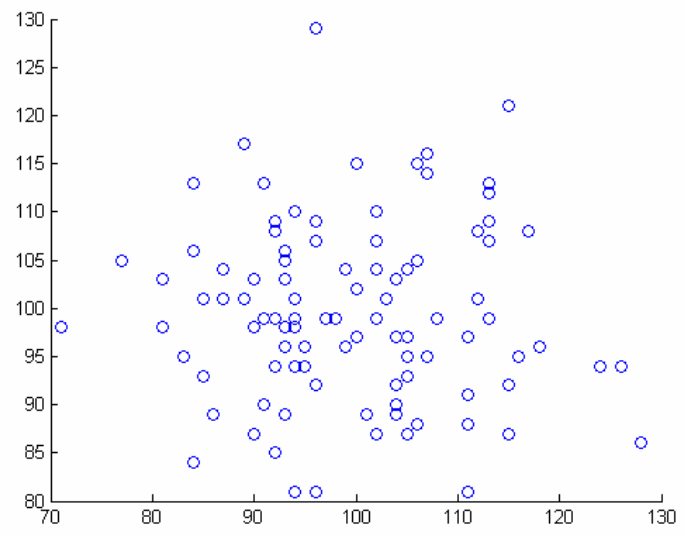

(c)
$\mathrm{P}_{\mathrm{t}}=10 \mu \mathrm{W}(\mathrm{b})$.

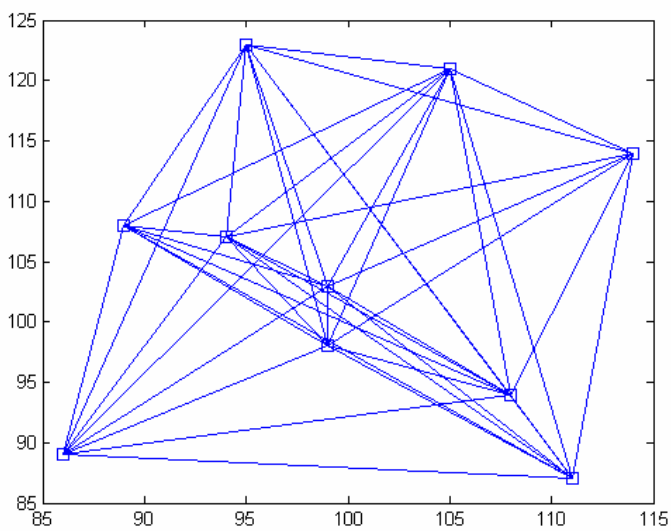

(b)

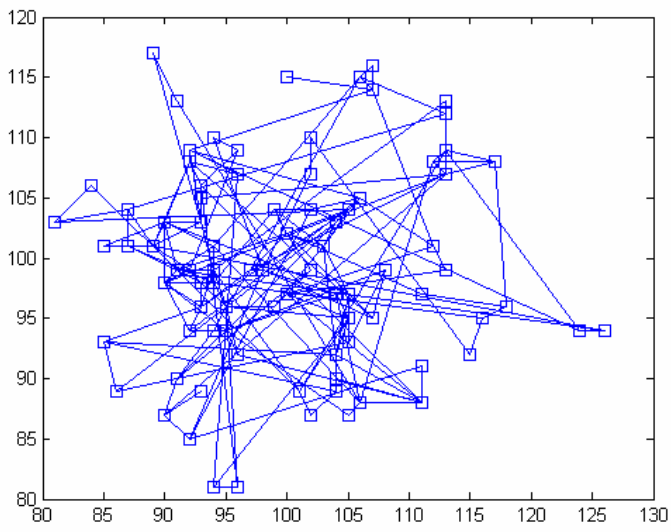

(d)

Fig. 3. Topology and available connections for different configurations of the network parameters: $N=10, p=0.1, P_{t}=10 \mathrm{~mW}(a-b), N=100$, $\mathrm{p}=0.05, \mathrm{P}_{\mathrm{t}}=10 \mu \mathrm{W}(\mathrm{c}-\mathrm{d})$. The other parameters are $\mathrm{N}_{0}=10^{-6}, \alpha=3, \beta=1$.

Figure 2 depicts the average number of connections per node using fixed transmission power and variable probability of accessing the medium $p$. The different power levels employed in the experiments are used to simulate two situations: (a) high transmission power, with mainly singlehop connections; (b) low transmission power, with multi-hop paths. Connectivity is achieved in both cases for low values of $p$, with a sharper behaviour in case (a) - deriving from the impact of collisions even by far nodes.

Furthermore, from the analysis of Fig. 2, it is possible to find an approximate value of the percolation threshold on the basis of the number of nodes in the network. Table I summarizes the empirical evaluation of the threshold value $p^{*}$ that enables network connectivity.

It can be noticed that in the case presented in fig. $2-a$, the critical value of $p$ that enables connectivity decreases as the number of nodes (and consequently collisions) increases, while in case (b) the behaviour is different, due to the fact that the range of interference is much more reduced.

For more clarity, figure 3 presents a snapshot of the node topology and the corresponding available links.
The proposed framework allows evaluation of the impact of several transmission and medium access control parameters on the performance of the ad-hoc network. In the following paragraphs, performance of the network in terms of normalized end-to-end capacity (capacity divided by occupied bandwidth) and bit error rate are presented. For sake of generality, results are presented as function of the MAC-level factor of merit $g_{M A C}$ (simply referenced as ' $g$ ' for clarity).

TABLE I. THRESHOLD VALUE $P^{*}$ FOR ACHIEVING CONNECTIVITY.

\begin{tabular}{|c|c|}
\hline No. of nodes & Critical value of $\boldsymbol{p}$ \\
\hline $10, P_{t}=10 \mathrm{~mW}$ (fig. $\left.2 a\right)$ & $>0.3$ \\
\hline $30, P_{t}=10 \mathrm{~mW}$ (fig. $\left.2 a\right)$ & $0.1<p^{*}<0.15$ \\
\hline $50, P_{t}=10 \mathrm{~mW}$ (fig. $\left.2 a\right)$ & 0.1 \\
\hline $100, P_{t}=10 \mathrm{~mW}$ (fig. $\left.2 a\right)$ & $0.05<p^{*<0.1}$ \\
\hline $10, P_{t}=10 \mu W$ (fig. $\left.2 b\right)$ & $0.05<p^{*}<0.1$ \\
\hline $30, P_{t}=10 \mu W$ (fig. $\left.2 b\right)$ & $0.15<p^{*}<0.2$ \\
\hline $50, P_{t}=10 \mu W$ (fig. $\left.2 b\right)$ & $0.05<p^{*}<0.1$ \\
\hline $100, P_{t}=10 \mu W$ (fig. $\left.2 b\right)$ & $0.05<p^{*}<0.1$ \\
\hline
\end{tabular}

Figure 4 depicts the normalized end-to-end capacity or spectral efficiency [bits/sec.Hz] (i.e. the end-to-end capacity 
per unit of frequency) for different levels of the signal-tonoise ratio at the receiver. In the figure, the $\mathrm{x}$-axis represents the ratio between the received power and termal noise, which is considered fixed, while the impact of interference by other nodes and merit of MAC strategy are considered in different curves. The ideal case considers perfect scheduling in access to the shared resource, which neglects mutual interference among neiboring nodes and brings to higher capacity; completely random access is the worst case, where no coordination among nodes occurs. The figure underlines that capacity variation is at maximum $20 \%$. Furthermore, as a comparison, capacity is reported in the case of fixed TDMA (Time Division Multiple Access), where resource are equally divided among 2 and 4 users in the same neighborhood. Reduced performance in spectral efficency is the price for complete and rigid scheduling and resource sharing - leading to perfect coordination in medium access and no interference.

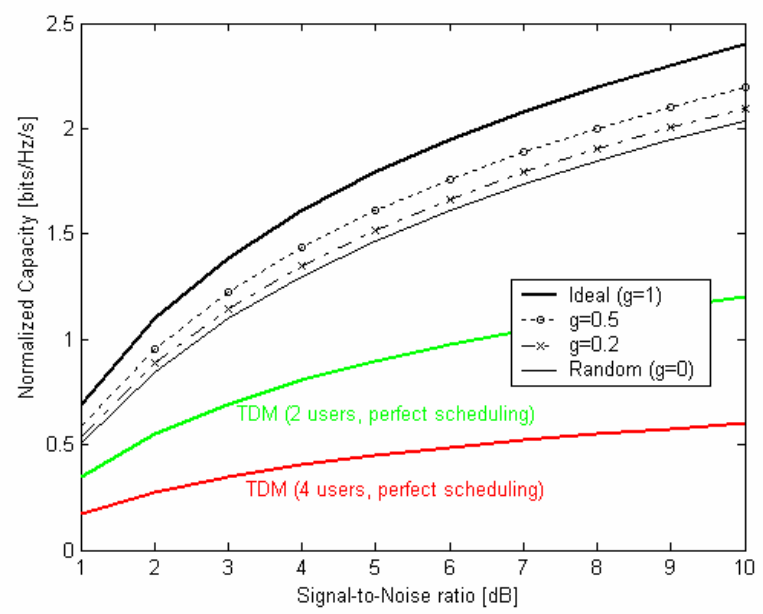

Fig. 4. Normalized capacity of the network for different signal-tonoise ratios and different MAC performance.

Fig. 4 allows to conclude that - in the considered scenario the factor of merit of the selected MAC strategy does not impact much on the end-to-end capacity of the ad-hoc network (around $20 \%$ at most), while the philosophy employed by the MAC protocol is more relevant: resource reservation / preassignment (like in TDMA) bring less advantage than contention-based solutions (upper curves).

Figure 5 provides an interesting insight on the impact of the MAC factor of merit on the BER performance of the multihop network. By using Eq. (23) it is possible to underline that the employment of a reliable medium access control strategy can dramatically improve the performance of the ad-hoc network, even of several orders of magnitude.

Summarizing, the MAC factor of merit (or probability of accessing the medium, in the considered slotted-ALOHA case) jointly with physical transmission parameters has a strong impact on the connectivity properties of the resulting ad-hoc network (see fig.2 and table I). This is due to the critical trade-off between transmission range and interference control, which suggest the usage either of a reliable MAC strategy and wider range, or a less complex MAC but also shorter range.

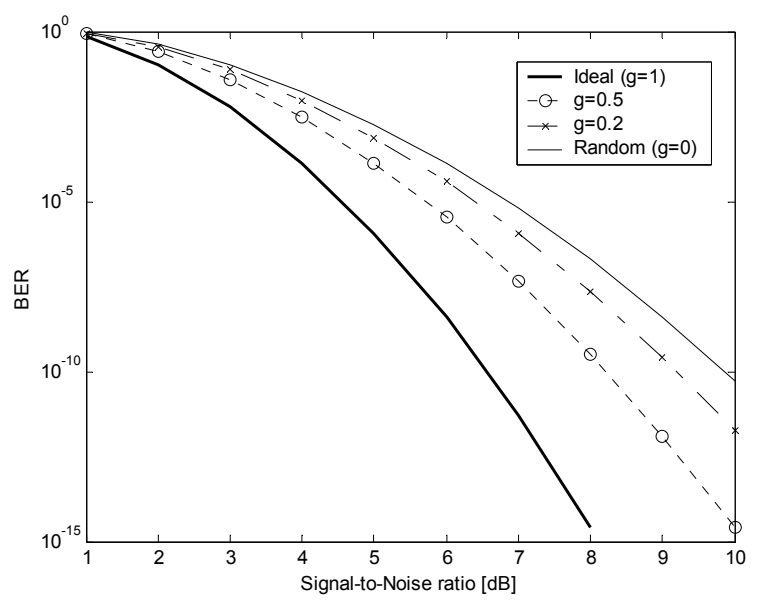

Fig. 5. BER performance against signal-to-noise ratio for different levels of MAC performance.

Another relevant outcome of the proposed numerical analysis is that MAC performance has limited impact in the end-to-end capacity of the network, while it dramatically affects transmission performance in terms of the bit error ratio.

\section{CONCLUSIONS AND FUTURE WORK}

The paper introduced a framework for the theoretical analysis of connectivity and performance issues in a wireless ad-hoc network. The application of the percolation theory jointly with communication theory principles allowed to derive some relevant and general analytical relationships among the network parameters, and the corresponding performance bounds in several scenarios. The paper presents the application of such framework in the case of a slottedALOHA medium access control strategy. Numerical analysis of the derived relationships and simulations are used in order to derive design principles for ad-hoc networks that consider the impact of physical and MAC-level parameters on network connectivity and end-to-end performance. Main outcome, shown through graphical comparisons, is that the MAC factor of merit (i.e. the capability to coordinate medium access in a given neighborhood) has a dramatic impact on the bit error ratio, while in terms of spectral efficiency the higher gain depends on using contention rather than scheduling in medium access.

Future work will be devoted in studying and introducing in the model additional MAC- and physical level characteristics, such as sub-optimal transmission performance, protocol overhead, and link-level retransmissions.

\section{REFERENCES}

[1] P. Gupta, P.R. Kumar, "The capacity of Wireless Networks," IEEE Trans. on Information Theory, Vol. 46, No. 2, pp. 388-404, March 2000. 
[2] O. Dousse, F. Baccelli, P. Thiran, "Impact of Interferences on Connectivity in Ad Hoc Networks," INFOCOM 2003, pp. 17241733.

[3] G. Grimmett, "Percolation," Springer, 1999.

[4] W. Stallings, "Data and Computer Communications," MacMillan, VI ed., 2003.

[5] A.B. Carlson, "Communication Systems," McGraw-Hill, Chapter 15, pp. 585-587.

[6] G. Ferrari, O.K. Tonguz, "Performance of Ad Hoc Wireless Networks with Aloha and PR-CSMA MAC Protocols," GLOBECOM 2003, San Francisco (U.S.A.), pp. 2824-2829.

[7] Mathworks, http://www.mathworks.com

[8] IETF Manet charter, http://www.ietf.org/html.charters/manetcharter.html

[9] Hongqiang Zhai, Jianfeng Wang, Xiang Chen, and Yuguang Fang, "Medium Access Control in Mobile Ad Hoc Networks: Challenges and Solutions," WCMC 2005, 2005.

[10] P. Karn, "MACA: a new channel access method for packet radio," 9th Computer Networking Conference, pp. 134-140, ARRL/CRRL Amateur Radio, 1990.

[11] C. L. Fullmer and J. Garcia-Luna-Aceves, "Floor acquisition multiple access (FAMA) for packet-radio networks," SIGCOMM '95, pp. 262-273. ACM, August 1995. Cambridge, MA (USA).

[12] V. Bharghavan, A. Demers, S. Shenker, and L. Zhang, "MACAW: A media access protocol for wireless LAN's," SIGCOMM '94, pp. 212-225. ACM, 1994.

[13] F. Talucci and M. Gerla, "MACA-BI (MACA by invitation): A receiver oriented access protocol for wireless multihop networks," PIMRC '97, Helsinki (Finland).
Fabrizio Granelli was born in Genoa in 1972. He received the «Laurea» (M.Sc.) degree in Electronic Engineering from the University of Genoa, Italy, in 1997, with a thesis on video coding, awarded with the TELECOM Italy prize, and the Ph.D. in Telecommunications from the same university, in 2001. Since 2000 he is carrying on his teaching activity as Assistant Professor in

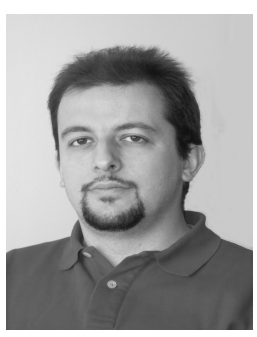
Telecommunications at the Dept. of Information and Communication Technology University of Trento (Italy). In August 2004, he was visiting professor at the State University of Campinas (Brasil). He is author or co-author of more than 45 papers published in international journals, books and conferences. Dr. Granelli is guest-editor of ACM Journal on Mobile Networks and Applications, special issue on "WLAN Optimization at the MAC and Network Levels" and CoChair of 10th IEEE Workshop on Computer-Aided Modeling, Analysis, and Design of Communication Links and Networks (CAMAD'04). Dr. Granelli is General Vice-Chair of the First International Conference on Wireless Internet (WICON'05) and General Chair of 11th IEEE Workshop on Computer-Aided Modeling, Analysis, and Design of Communication Links and Networks (CAMAD’06). 